\title{
Relativistic Precessions and Classical Electrodynamics
}

\section{Hajra S.}

Calcutta Mathematical Society, India;

E-mail: Hajra <sankarhajra@yahoo.com>;

Electric charges possess momenta and energies that we could experience with our sense organs. Therefore, electric charges are real physical entities i.e., objects. All objects are subject to gravitation. Electric charges should similarly be the subject to gravitation. The acceleration of a point charge should, therefore, be the same as that of a point object in magnitude and in direction in the same gravitating field and that acceleration, too, should be directed towards the interacting gravitating field. This implies that the gravitating mass of a point charge is proportional to its longitudinal electromagnetic mass $\gamma^{3} m_{0}$ (where $\gamma=1 / \mathrm{k}$ and $\mathrm{k}=\left(1-\mathrm{u}^{2} / \mathrm{c}^{2}\right)^{1 / 2}$, $\mathrm{u}$ the velocity of the charge in the free space and $\mathrm{m}_{0}$ is the rest electromagnetic mass of the charge). We shall show in this paper that this simple classical consideration along with classical physics is equivalent to the general relativity theory.

Keywords: Classical electrodynamics, De Sitter precession, Lense-Thirring precession, precession of perihelion of Mercury, bending of light rays, gravitational red shift, Thomas Precession.

DOI: $10.18698 / 2309-7604-2015-1-199-212$

\section{Introduction}

In the previous discussion [1], we have used the classical consideration that electric and magnetic fields possess momenta and energies that we could experience with our sense organs. Therefore, these are real physical entities i.e., objects. All objects are subject to gravitation and at the near vicinity of the Earth's surface they are carried with the Earth. Therefore, electric and magnetic fields should similarly be subject to gravitation and at the vicinity of the Earth's surface they should similarly be carried with the Earth. We have shown therein that this simple classical consideration along with classical physics is equivalent to the special relativity theory.

In the present discussion, we shall show that classical physics along with a similar classical consideration that electric charges are subject to gravitation is equivalent to the general relativity theory.

To reach such an interesting conclusion let us review three electrodynamic quantities viz,. Electromagnetic momentum (P), Longitudinal electromagnetic mass (LEM) and Transverse electromagnetic mass (TEM) relating to a steadily moving point charge. 


\section{Three classical Electrodynamic Equations}

Following Maxwell-Heaviside, Searle elegantly deduced the magnetic energy (T) of a steadily moving point charge. Following Searle, we have deduced the electromgnetic momentum (P), Longitudinal electromagnetic mass (LEM) and Transverse electromagnetic mass (TEM) classically in the previous discussion [1] and elsewhere [2, 3] which are as follows:

$$
\begin{gathered}
\mathbf{P}=\gamma \frac{Q^{2} \mathbf{u}}{6 \pi \varepsilon_{0} c^{2} \delta R}=\gamma m_{0} \mathbf{u}\left(m_{0}=\frac{Q^{2}}{6 \pi \varepsilon_{0} c^{2} \delta R}, k=\left(1-u^{2} / c^{2}\right)^{1 / 2}, \gamma=1 / k\right) \\
L E M=\gamma^{3} m_{0} \\
T E M=\gamma m_{0}
\end{gathered}
$$

$\mathrm{Q}$ is the quantity of charge associated with an extremely small conducting sphere with radius $\delta \mathrm{R}$, $\mathbf{u}$ is the velocity of the charge .

All those three electromagnetic quantities are real physical quantities and they do exist due to the real existence of a point charge and its motion.

The equation of motion of a point charge is determined by those two real electromagnetic masses, i.e., Longitudinal and Transverse electromagnetic masses of a steadily moving point charge.

Now the question arises, if a point charge is subject to gravitation, what are the contributions of these real electromagnetic masses to the gravitating mass of a point charge when the point charge steadily moves in a gravitating field?

We shall study the problem in the next sections.

\section{Gravitating and inertial masses of electric charges}

Electric charges possess momenta and energies that we could experience with our sense organs. Therefore, these too are real physical entities i.e., objects. All objects are subject to gravitation. Electric charges should similarly be subject to gravitation.

The acceleration of a point charge should, therefore, be the same as that of a point object in magnitude and in direction in the same gravitating field and that acceleration, too ,should be directed towards the interacting gravitating field (unlike its acceleration during its interaction with 
the electric field). This implies that the gravitating mass of a point charge is proportional to its longitudinal electromagnetic mass $\gamma^{3} m_{0}$.

Transverse electromagnetic mass $\gamma m_{0}$ should play no role in this interaction. If it played any role in this interaction, material bodies (all of which contain charges) should have acceleration not always directed towards the centre of gravity of the gravitating body and that acceleration too would differ in magnitude and in direction in different interactions. But this is not the case as observed in many precise experiments. Thus, in a gravitating field, a point charge acts as a mass point; mass of the mass point is proportional to the longitudinal electromagnetic mass of the point charge.

Suppose that an object of material mass $m_{m}$ contains ' $Q$ ' amount of positive and negative charges with the rest electromagnetic mass $m_{0}$ in total [vide Eqs. (1) \& (2)] and for simple calculation assume that the positive and negative charges are concentrated at two points separately near the centre of mass of the object. Therefore, an object (containing charges) at rest in free space should have two masses viz., material mass $m_{m}$ and electromagnetic mass $m_{0}$, both of which are subject to gravitation.

But we do know neither the material mass $m_{m}$ nor the rest electromagnetic mass $m_{0}$ associated with the object. We know only

$$
\left(m_{m}+m_{0}\right)=m
$$

which is a measurable quantity measured when the object is at rest on the surface of the Earth.

When the velocity is large, mass of the object should be $m_{m}+\gamma^{3} m_{0}$ which is not a determinable quantity and, therefore, could not be used for experimental physics. In any case it could not be greater than $\gamma^{3}\left(m_{m}+m_{0}\right)=\gamma^{3} m$ which is a determinable quantity and, therefore, we could use it as the limiting gravitating mass of an object moving with high velocity. This limiting value of the gravitating mass of an object will help us to understand the approximate motion of objects in a gravitating field.

Thus, we see that the limiting gravitating mass of an object (LGM)

$$
L G M=\gamma^{3}\left(m_{m}+m_{0}\right)=\gamma^{3} m
$$


and if Galileo's experiment was exactly valid for all objects, this mass is proportional to the inertial mass of the object.

Therefore, considering the proportionality constant to be unity, we could write that the limiting inertial mass (LIM) of the object is

$$
L I M=\gamma^{3}\left(m_{m}+m_{0}\right)=\gamma^{3} m
$$

which implies that the linear momentum (P) of the object is [3]

$$
\boldsymbol{P}=\gamma^{3}\left(m_{m}+m_{0}\right) \boldsymbol{u}=\gamma^{3} m \boldsymbol{u}
$$

\subsection{Equation of motion of the planets round the Sun}

Let us now study the motion of a planet (which obviously contains charges) with an initial velocity u having limiting mass $\gamma^{3} \mathrm{~m}$ [as per Eqs. (4), (5) and (6)] when it passes through the gravitating field of the sun having mass $\mathrm{M}$ (material mass + mass originating from associated charges). The subsequent motion of the planet will be confined to the plane containing the direction of acting force and the direction of initial velocity. Let us fix a polar coordinate in this plane where the centre of the sun is the origin and the initial position of the planet is $(r, \theta)$.

The motion of this planet as per Newtonian physics should be governed by following equations.

\section{Radial Force}

$$
-\gamma^{3} G M m / r^{2}=\gamma^{3} m\left(\ddot{r}-r \dot{r} \dot{\theta}^{2}\right)
$$

where $\mathrm{G}$ is the gravitational constant and the Cross-Radial Force

$$
\begin{gathered}
\frac{1}{r} \frac{d}{d t}\left(\gamma^{3} m \times r^{2} \dot{\theta}\right)=0 \\
\gamma^{3} r^{2} \dot{\theta}=H=\text { cons } \tan t
\end{gathered}
$$


where $\mathrm{m}$ has been replaced by $\gamma^{\mathbf{3}} \mathrm{m}$ as per our analysis in Section (3).

Using Eqs. (9) and (10) we have for a very small eccentricity of the planets [3]

$$
\frac{d^{2} U}{d \theta^{2}}+U \approx \frac{G M}{H^{2}} \gamma^{6} \approx \frac{G M}{H^{2}}+\frac{3 G M}{c^{2} r^{2}}
$$

\subsection{Equation of Motion of the light rays in the gravitating field of the Sun}

Light-rays possess electromagnetic momentum and electromagnetic energy. Therefore, a point light will similarly be subject to gravitation as in the case of a point charge. But in this case, for a point light $m_{0} \rightarrow 0$, and, therefore, $\mathrm{H} \rightarrow \propto$ and the equation of motion of a point light in a gravitating field will be following Eq. (11)

$$
\frac{\mathrm{d}^{2} \mathrm{U}}{\mathrm{d} \theta^{2}}+\mathrm{U} \approx \frac{3 \mathrm{GM}}{\mathrm{c}^{2} \mathrm{r}^{2}}
$$

when light moves through a medium near the sun or when light from a star has lost much of its velocity as it moves away from the star. This will at once explain the bending of light rays grazing the surface of the sun.

\section{Gravitational Red Shift}

Similarly, a ray coming from a distant star will lose its velocity due to the gravitating force of the star acting on the ray and thereby the frequency of the ray will change as it moves away from the star. The abridged deduction is given below.

Suppose that a ray with the radian frequency $\omega$ is coming from the surface of a star of radius $R$ and of mass $M$ to the surface of the Earth which is $x$ distance away from the centre of a star. As per our previous discussion, electromagnetic energy has the same acceleration as that of material bodies as well as point charges in the same gravitational field.

Let $f(R)$ be the gravitational acceleration of a ray on the surface of a star and $\mathbf{f}(\mathrm{x})$ be the gravitational acceleration of the same ray when it is on the surface of the Earth.

Then, we have from the law of gravitation [4], 


$$
\frac{f(x)}{f(R)}=\frac{R^{2}}{x^{2}} \quad \text { where } f(x)=\frac{G M}{x^{2}}
$$

Now, we have the differential equation for the velocity of the ray,

$$
\int_{c}^{v} \frac{d V}{d x}=-G M \int_{R_{t}}^{x} \frac{d x}{x^{2}}
$$

where ' $c$ ' is the velocity of the ray on the surface of the star and $v$ is the velocity of the same ray on the surface of the Earth. From which we have,

Therefore,

$$
v=c\left(1-\frac{G M}{R c^{2}}\right)
$$

when $x$ is large, from which we have,

$$
\omega^{\prime} / \omega=\left(1-\mathrm{GM} / \mathrm{Rc}^{2}\right)
$$

( $\omega^{\prime}$ is the radian frequency of the same light ray at the surface of the Earth), as the number of complete waves passing through a point (i.e., frequency) must be proportional to the velocity of the wave. For full deduction vide [2].

\section{Precessions of orbiting spinning gyroscopes and orbiting rigid spinning electrons}

(a) Orbital Precession [3,5]

Precession is literally the change in the orientation of the axis of the spinning body. It is measured by the angle between the initial position and the final position of the spinning axis of the gyroscope.

When a spinning gyroscope is rotating in a near-Earth, near-circular polar orbit around the Earth, apart from spinning, the gyroscope has two other motions, viz., (i) the significant driving motion with a velocity $u$ in the orbital plane due to its motion in its orbit and (ii) an insignificant Coriolis motion with an acceleration $-2 \omega_{\mathbf{E} u \sin \varphi}\left(\omega_{\mathbf{E}}\right.$ is the angular velocity of the spinning Earth, $\varphi$ is the latitude at the point of observation) due to the Coriolis action of the 
spinning Earth. We shall show below that each motion will cause precessions in each relevant plane.

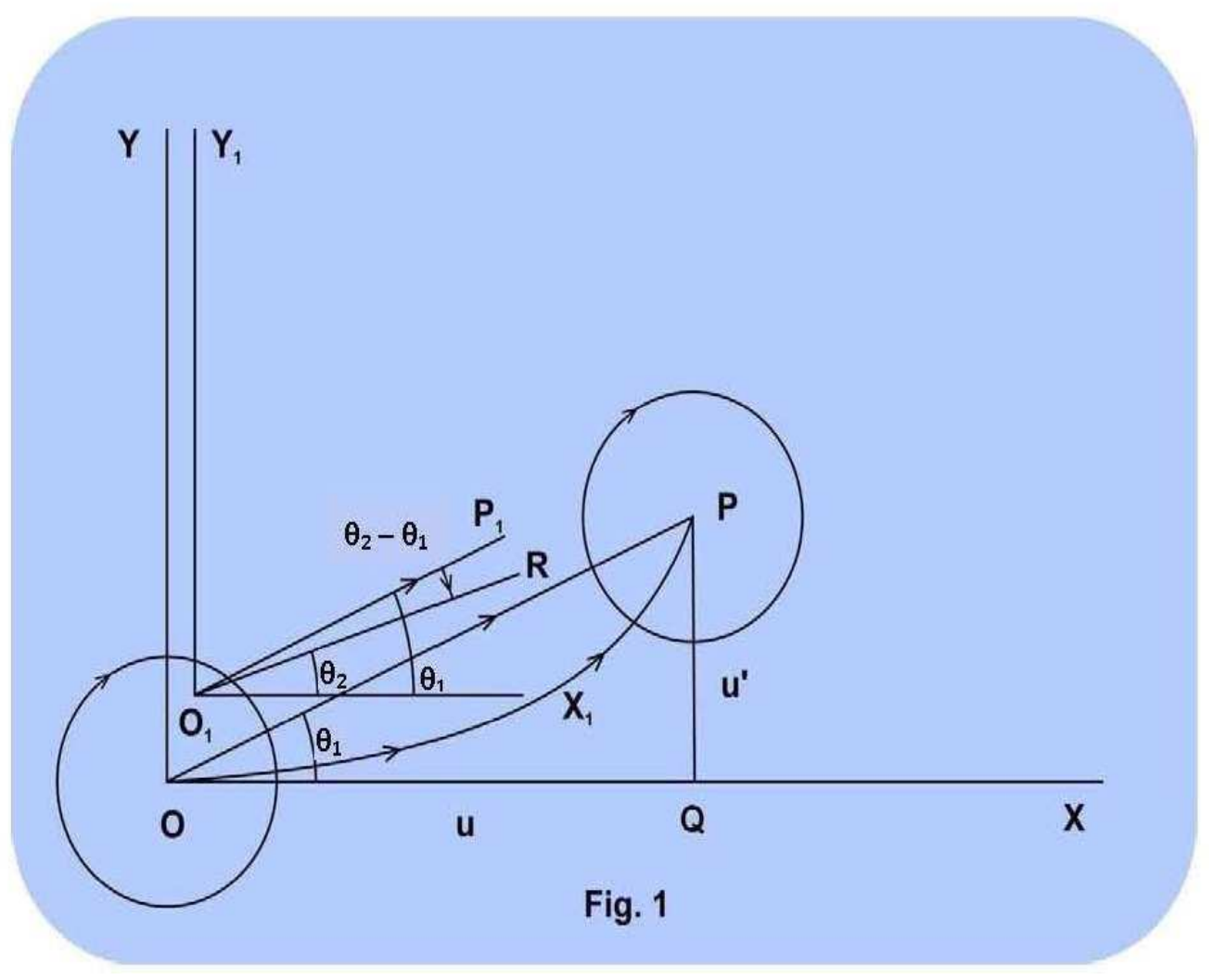

Fig. 1. Orbital precession of an orbiting spherical rigid spinning gyroscope

(The brief outline of deduction: When a gyroscope moves in its near-Earth near -circular orbit around the Earth, in the orbital plane (motion plane) as well as in tangential plane (Coriolis plane), the direction of momentum of any infinitesimal mass element of the gyroscope should be different from the constrained direction of movement of that mass element carried with the centre of the gyroscope. This difference of directions of motion of the same mass element at the same instant should cause precessions of the gyroscope in the motion plane as well as in the Coriolis plane.)

Now consider that this paper plane is the orbital plane of the orbiting gyroscope. The centre of the gyroscope starts its orbital motion with a velocity $\mathrm{u}$ from the point $\mathrm{O}$ and in an infinitesimal time interval $d t$ it reaches the point $\mathrm{P}$. We may consider roughly that during the time interval $d t$, the path OP is a small straight line. The motion of the centre of the gyroscope in the orbital plane is a curved motion, and therefore, that motion is the resultant of two motions i.e., (i) one significant motion $\mathrm{u}_{\mathbf{x}}$ towards $\mathrm{OX}$, tangent to the orbit at $\mathrm{O}$ and (ii) an insignificant motion $\mathrm{u}_{\mathbf{y}}$ towards OY normal to the tangent at O (Fig.1). 
Therefore, the angle between the direction of motion of the centre of the gyroscope and OX

$$
\tan X O P=\tan \theta_{1}=\frac{u_{y}}{u_{x}} \approx \frac{u^{\prime}}{u}
$$

In circular motion, the tangential component is much more than the normal component, and in the instant case $\theta_{1}$ is extremely small, therefore we have

$$
\theta_{1}=\frac{u^{\prime}}{u}
$$

Now the x-component and the y-component of the linear momentum of the infinitesimal mass element of the moving gyroscope body around any point $O_{1}$ in the orbital plane could be written as per Eq. (7)

$$
\begin{aligned}
& P_{x}=\gamma^{3} m u \\
& P_{y}=m u^{\prime}
\end{aligned}
$$

where $u$ is large, $u^{\prime}$ is very small, $m$ is the limiting gravitating (or inertial) mass element of the infinitesimal body element of the gyroscope at rest in free space as defined in Eq. (4) .

Therefore, from Eq. (20), we find that the resultant direction $\left(O_{1} R\right)$ of momentum of that infinitesimal mass element at $O_{1}$ will make an angle $\theta_{2}$ with $O_{1} X_{1}$ axis drawn parallel to $\mathrm{OX}$ at $O_{1}$ such that

$$
\theta_{2}=\frac{P_{y}}{P_{x}}=k^{3} \frac{u^{\prime}}{u}
$$

But, the infinitesimal mass element of the gyroscope body at $O_{1}$ is constrained to move with the centre of the gyroscope towards $O_{1} P_{1}$ parallel to OP such that 


$$
\angle X_{1} O_{1} P_{1}=\theta_{1}=\frac{u^{\prime}}{u}
$$

Now the centre of the gyroscope is constrained to move in its orbit, but the axis of the gyroscope could tilt in any direction. Therefore, infinitesimal body element of the gyroscope near the point $\mathrm{O}_{1}$ will be displaced towards $\mathrm{O}_{1} \mathrm{X}_{1}$ parallel to $\mathrm{OX}$ in the orbital plane. If this displacement does not change the angular momentum of the spinning gyroscope, the angular displacement in the orbital plane could be measured by

$$
d \theta=\theta_{2}-\theta_{1}=\left(k^{3}-1\right) \frac{u^{\prime}}{u}
$$

Now in the case where OP is extremely small, we may consider that the magnitudes of both the velocity components of the instantaneous motion of the spinning orbiting body at the points $\mathrm{O}$ and $\mathrm{P}$ are roughly the same. Therefore, at the point $\mathrm{P}$, the velocity components of the motion of the centre of the electron read

$$
\begin{aligned}
& u_{x}=u=\omega_{g} r \cos \delta \\
& u_{y}=u^{\prime}=\omega_{g} r \sin \delta
\end{aligned}
$$

where $\delta$ is the angle that the arc OP subtends at the centre (not shown in (Fig.1) and $\omega$ is the angular velocity of the orbiting gyroscope in its orbit having radius $r$, from which we get

$$
\tan \delta \approx \frac{u^{\prime}}{u} \approx \frac{u d t}{r}
$$

In case the spinning axis of the gyroscope is not normal to the orbital plane, it should precess in the orbital plane with angular velocity 


$$
\frac{d \theta}{d t}=\left(\Omega_{g}\right)_{O}=-\frac{3}{2} \frac{u^{2}}{c^{2}} \frac{u}{r}=-\frac{3}{2} \frac{u^{2}}{c^{2}} \omega_{g}
$$

where $\omega_{\mathrm{g}}$ is the angular velocity of the gyroscope in its orbit.

In case of an orbiting rigid spinning electron, the momentum equation is Eq. (1) instead of the Eq. (7) and therefore, in that case precession velocity of the gyroscope in the orbital plane should be

$$
\frac{d \theta}{d t}=\left(\Omega_{e}\right)_{O}=-\frac{1}{2} \frac{u^{2}}{c^{2}} \frac{u}{r}=-\frac{1}{2} \frac{u^{2}}{c^{2}} \omega_{e}
$$

where $\omega_{\mathbf{e}}$ is the angular velocity of the electron in its orbit.

(b) Tangential precession $[3,5]$

In the tangential plane (Fig. 2), the centre of the gyroscope is moving towards the North $\mathrm{ON}$ with a velocity $\mathrm{u}$. The Coriolis average velocity $(0+2 \omega \mathrm{Eu} \sin \varphi \mathrm{dt}) / 2=\omega \mathrm{Eu} \sin \varphi \mathrm{dt}$ is towards the East. Carried with the centre of the gyroscope, the resultant direction of motion of an infinitesimal mass element of the gyroscope near the point $\mathrm{O}_{1}$ makes an angle $\theta_{1}$ with $\mathrm{O}_{1} \mathrm{~N}_{1}$ parallel to the North $\mathrm{ON}$ in the tangential plane such that

$$
\theta_{1}=\frac{u^{\prime}}{u}=\frac{\omega_{E} u(\sin \varphi) d t}{u}=\omega_{E}(\sin \varphi) d t
$$




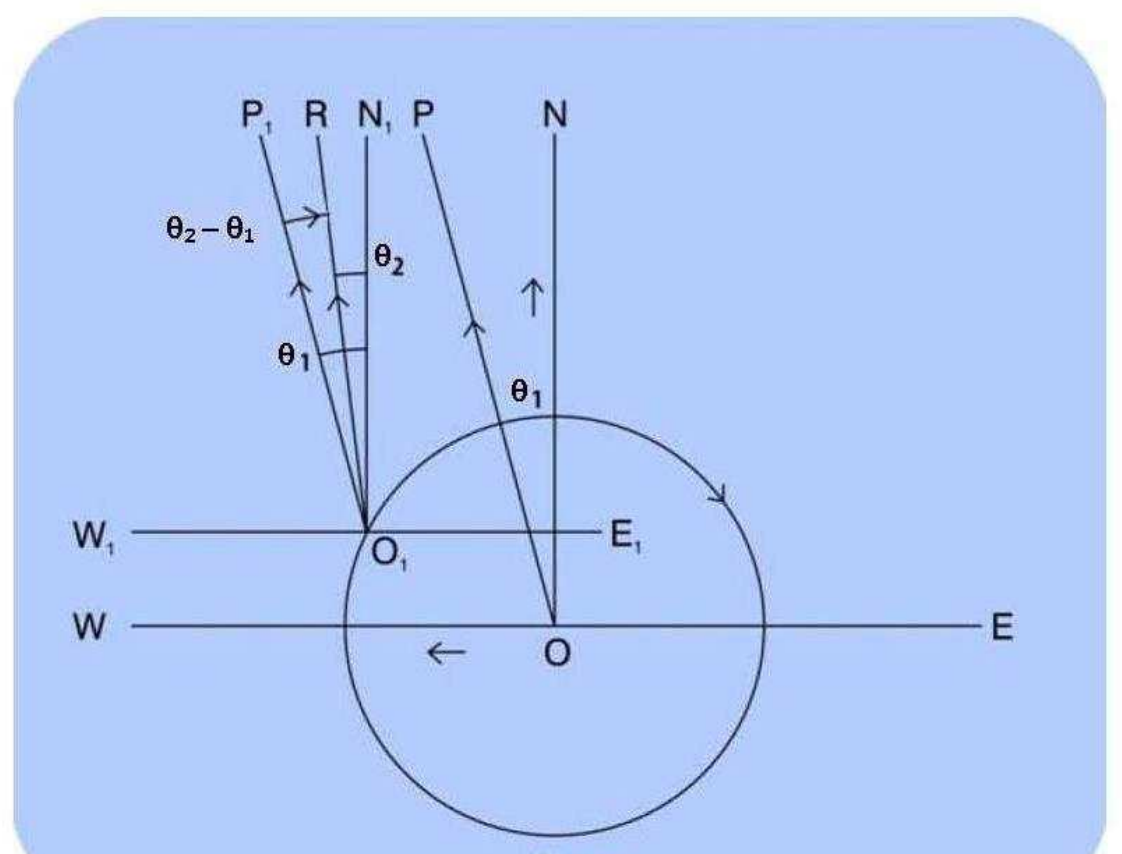

Fig 2

Fig 2. Tangential (Coriolis) Precession of an orbiting spherical rigid spinning gyroscope.

Now the $\mathrm{x}$ - component (towards $\mathrm{O}_{1} \mathrm{~N}_{1}$ ) and the $\mathrm{y}$ - components (towards $\mathrm{O}_{1} \mathrm{~W}_{1}$ ) of the momentum of that infinitesimal mass element of the moving gyroscope around the point $O_{1}$ in the tangential plane could be written as per Eq. (7) as given in Eq. (20)

$$
P_{x}=\gamma^{3} m u \quad P_{y}=m u^{\prime}
$$

Therefore, the resultant direction of momentum of that infinitesimal mass element of the gyroscope near the point $\mathrm{O}_{1}$ will make an angle $\theta_{2}$ with $\mathrm{O}_{1} \mathrm{~N}_{1}$ parallel to $\mathrm{ON}$, the North in the tangential plane such that

$$
\theta_{2}=\frac{P_{y}}{P_{x}}=k^{3} \frac{u^{\prime \cdot}}{u}=k^{3} \omega_{E}(\sin \phi) d t
$$

Therefore, infinitesimal mass element of the gyroscope near the point $\mathrm{O}_{1}$ will be displaced in the tangential plane towards $\mathrm{O}_{1} \mathrm{~N}_{1}$ parallel to $\mathrm{ON}$, the North. If this displacement does not 
change the angular momentum of the spinning gyroscope, the angular displacement in the tangential plane could be measured by

$$
\theta_{2}-\theta_{0}=d \theta=\left(k^{3}-1\right) \omega_{E}(\sin \varphi) d t=-\frac{3}{2} \omega_{E}(\sin \varphi) d t
$$

In case the axis of the gyroscope is not normal to the tangential plane, the gyroscope will precess in tangential plane with the angular velocity

$$
\frac{d \theta}{d t}=\left(\Omega_{g}\right)_{T}=-\frac{3}{2} \omega_{E}(\sin \varphi)
$$

The magnitude and direction of this precession change with latitude. When averaged over the orbit [3], the result is equatorial precession as per the following equation

$$
\left\langle\left(\Omega_{g}\right)_{T}\right\rangle=\left(\Omega_{g}\right)_{E q}=-\frac{3}{4} \frac{u^{2}}{c^{2}} \omega_{\mathrm{E}}
$$

The magnitude of Coriolis acceleration may decrease by some factor $\kappa$ (averaged over the orbit) which should originate from the environmental and experimental conditions that the moving gyroscope faces in its orbit. The Earth possesses magnetic field and it should have some contribution to the precession of the gyroscope as the gyroscope contains charges and currents and shielding could not eliminate the full effect. Therefore, Eq. (33) should now be changed to

$$
\left\langle\left(\Omega_{g}\right)_{T}\right\rangle=\left(\Omega_{g}\right)_{E q}=-\frac{3}{4} \kappa \frac{u^{2}}{c^{2}} \boldsymbol{\omega}_{\mathrm{E}}+\mathbf{a}
$$

Where $\mathbf{a}$ is the contribution to precession due the residual magnetic field of the Earth affecting the gyroscope. 


\section{The Experiments of Michelson-Gale and Bilger et al.}

The Earth carries electromagnetic fields along with it and thereby light at the vicinity of its surface should be affected by the Coriolis force due to the spinning of the Earth .

Let us choose a point $\mathrm{O}$ with the latitude $\alpha^{0}$ North and construct a tangential plane at this point. Now let us fix a Cartesian co-ordinate system in the plane such that OY represents the North and OX represents the East. Now suppose that the Earth is not spinning and an element of light beam is arranged to move from a point $\mathrm{P}$ in the $\mathrm{OY}$ axis at the instant $t=0$ in a small circular motion in the clockwise direction such that at the time $t$ it touches the point $\mathrm{Q}$ in the $\mathrm{OX}$ axis and say $O P=O Q=r$. That is when $t=0, x=0, y=r$ and when $t=t, x=r, y=o$.

Now suppose that the Earth spins with an angular velocity $\Omega$. Then the Coriolis force due to the spinning of the Earth should deflect the beam mainly eastwardly and the beam will not touch the point $\mathrm{Q}$. Instead it will touch a point $\mathrm{R}$ very adjacent to the $\mathrm{OX}$ axis. Now for a rough calculation of the distance OR, let us consider the motion of the beam on the OY axis with a velocity ' $c$ ' from the point $\mathrm{P}$ to the point $\mathrm{O}$ directly. In this case, Coriolis Force $\mathrm{F}_{\mathbf{x}}$ is acting on the beam and so we may write,

$$
F_{x}=\frac{d^{2} x}{d t^{2}}=-2 \Omega(-c) \sin \alpha=2 \Omega \sin \alpha
$$

Remembering the initial condition $\mathrm{t}=\mathrm{r} / \mathrm{c}$, we have,

$$
x=\Omega r^{2} \sin \alpha / c
$$

This means that the beam will be deflected towards east by the amount $\mathrm{x}$ as per Eq.(36). Now Let the beam be divided at $\mathrm{P}$ in two parts and each part is made to travel in clockwise and anticlockwise directions in a circular path of radius $\mathrm{r}$ to meet at $\mathrm{P}$ again. Due the Coriolis action on the beams, the approximate radii of both the beams should be respectively

$$
\begin{gathered}
(O R=) r+\Omega r^{2} \sin \alpha / c \\
r-\Omega r^{2} \sin \alpha / c
\end{gathered}
$$

The Path difference $\quad 2 \pi\left(r+\frac{\Omega r^{2}}{c} \sin \alpha\right)-2 \pi\left(r-\frac{\Omega r^{2}}{c} \sin \alpha\right)=\frac{4 \Omega A}{c} \sin \alpha$

From the last two equations we have for one complete rotation 


$$
\begin{aligned}
\qquad \Delta t=\frac{4 \Omega A}{c^{2}} \sin \alpha \\
\text { Fringe shift }=\frac{4 A \Omega \sin \alpha}{c \lambda}
\end{aligned}
$$

where A is the area of the circle . Fringe shifts relating to Equation (44) seem to be verified by the Experiments of Michelson-Gale - Pearson and Bilger et al [6].

\section{Conclusion}

Our study shows that all general relativistic phenomena could easily and rationally be explained from the consideration of classical physics and thereby exposes the uselessness of the general relativity theory in the domain of gravitational physics.

Acknowledgement: Your author gratefully acknowledges the grant provided to him by Dr. M. C. Duffy, Founder- Secretary of the PIRT-London to present this paper personally in the PIRTMoscow 2015 conference

\section{References}

1. Hajra S. (2015). Classical Electrodynamics and the Special Relativity Theory. PIRT-Moscow.

2. Hajra S. (2012). Classical Interpretations of Relativistic Phenomena. Jour. Mod. Phy., 3 (2), 187-199.

3. Hajra S. (2014). Classical Interpretations of relativistic precessions, Chin, Phy. B 23 (4) 40402- (2014).

4. Starzhinskii V.M. (1982). An Advance Course of Theoretical Mechanics. Moscow: MIR [World], 264-265.

5. Everitt W.F.C. (2011). Gravity Probe B: Final Results of a Space Experiment to Test General Relativity. Phy. Rev. Lett., 106, 221101-5.

6. Anderson R. (1994). A Century of Earth Rotated Interferometer. Am. J. Phys., 62, 975-985. 